\title{
Pharmacokinetics of Ethylene in Man by On-line Laser Photoacoustic Detection
}

\author{
H.W.A. Berkelmans ${ }^{b}$ B.W.M. Moeskops ${ }^{b}$ J. Bominaar ${ }^{b}$ \\ P.T.J. Scheepers ${ }^{a}$ F.J.M. Harren ${ }^{\text {b,1 }}$ \\ ${ }^{a}$ Department of Epidemiology and Biostatistics, UMC St Radboud, Nijmegen, The \\ Netherlands \\ ${ }^{\mathrm{b}}$ Department of Molecular and Laser Physics, University of Nijmegen, Nijmegen, \\ The Netherlands \\ adress for manuscript correspondence: Bas Moeskops \\ basmoesk@sci.kun.nl \\ Fax: (+31)-24-3653311 \\ Telephone (+31)-24-3653029
}

\footnotetext{
1 To whom correspondence should be addressed at Department of Molecular and Laser Physics, University of Nijmegen, P.O. Box 9010, NL-6500 GL Nijmegen, The Netherlands.
} 
Pharmacokinetics of Ethylene in Man by On-line Laser Photoacoustic

Detection. H.W.A. Berkelmans, B.W.M. Moeskops, J. Bominaar, P.T.J.

Scheepers, F.J.M. Harren Toxicol. Appl. Pharmacol.

\begin{abstract}
The pharmacokinetics of ethylene are determined using laser based photoacoustic detection and a closed chamber setup. Concentration-time data are analyzed using a two-compartment and a physiologically based pharmacokinetic (PBPK) model. Endogenous production was $92 \pm 13 \mathrm{pmol} \cdot \mathrm{h}^{-1} \mathrm{~kg}^{-1}$ for the two-compartment model and $75 \pm 10 \mathrm{pmol} \cdot \mathrm{h}^{-1} \cdot \mathrm{kg}^{-1}$ for the PBPK model. These values agree with previous work at our department, but are significantly lower than published values based on gas chromatography. The blood:air partition coefficient in the PBPK model was determined by curve fitting, because simulations based on published values did not agree well with data. Curve fitting gave a value of $0.092 \pm 0.029$. The real-time nature and high sensitivity of photoacoustic detection make it a useful addition to gas chromatography in closed chamber studies.
\end{abstract}

Key words: photoacoustic spectroscopy, closed chamber technique, ethylene, human, breath, pharmacokinetics, ethene, free radicals, lipid peroxidation 


\section{Introduction}

Trace gas analysis of the composition of exhaled air gives information about a wide variety of processes occurring inside the human body. One such process is free-radical mediated lipid peroxidation. In lipid peroxidation, polyunsaturated fatty acids react with reactive oxygen species to form a variety of products, including pentane, ethane and ethylene (ET) (Esterbauer, 1996; Harren et al., 1999). In this study ET will be measured as a marker for this process. However, ET is not only produced during lipid peroxidation. Other suggested sources of endogenous ET are the oxidation of free methionine (Lieberman et al., 1964; Kessler and Remmer, 1990), oxidation of hemoglobin (Kessler, 1987) and metabolism of intestinal bacteria (Gelmont et al., 1981; Törnqvist et al., 1989). It was shown that ET produced by oxidation of methionine forms a minor and negligible contribution to total ET production (Kneepkens et al., 1994).

A previous study from our department suggested that the use of ET as a fast, non-invasive and on-line biomarker of lipid peroxidation is possible (Harren et al., 1999). Knowledge of the pharmacokinetic parameters of ET in healthy human subjects is a prerequisite to useful applications in humans. Studies of the pharmacokinetics of ethylene in man have been published previously (Csanády et al., 2000). Often pharmacokinetic parameters are determined from gas uptake studies, which conventionally use a closed chamber technique (Filser, 1992) combined with gas chromatography. This technique requires concentration of the hydrocarbons on a solid sorbent. The choice of the proper sorbent material during the concentration step is critical. Permeability of and adsorption to the wall of the sample container, and possible contamination with ambient air may cause artefacts which make breath samples uninterpretable. To overcome this delicate and time consuming concentration step we propose to use a laser based trace gas detection technique, which has such a high sensitivity for ET that accumulation is no longer needed, and online measurements become possible.

The goal of this study is to determine the pharmacokinetics of ET with this laser based detection and a closed chamber setup. From the data the parameters of both a two-compartment and a physiologically based pharmacokinetic (PBPK) model are determined. 


\section{Methods}

Exposure system

To investigate the pharmacokinetics of ET, a closed breath circuit was used (Fig.1). This circuit consists of 2 glass pots containing $\mathrm{CO}_{2}$-absorbing sodalime ${ }^{\circledR}$, a $2 \mathrm{~L}$. balloon, an $\mathrm{O}_{2}$-inlet, pressure valves and a mouthpiece. The $\mathrm{O}_{2}$-concentration was monitored by an infrared gas analyser (URAS 14, ABB, Zurich, Switzerland) and kept at $21 \pm 1 \%$ vol by addition of $100 \% \mathrm{O}_{2}$ using a $25 \mathrm{~L} \cdot \mathrm{h}^{-1}$ mass flow controller (5850S, Brooks Instrument, Veenendaal, The Netherlands). The closed breath circuit was connected to the detection system, the URAS 14 and the $\mathrm{O}_{2}$-bottle as shown in Fig. 1. The flow through the detection cell was kept at $3.00 \pm 0.01 \mathrm{~L} \cdot \mathrm{h}^{-1}$ by a membrane pump combined with a mass flow controller (5850S, Brooks Instrument). All flow rates were measured and controlled by a Read Out \& Control Interface (0154, Brooks Instrument).

\section{Real-time monitoring of ethylene}

The ET concentration was monitored in real time by using a sensitive laserbased photoacoustic detector. A schematic diagram of the setup is given in Fig. 1. Briefly, the detector consists of a line-tunable $\mathrm{CO}_{2}$-laser emitting radiation in the 9 to $11 \mu \mathrm{m}$ region and a photoacoustic cell, in which the gas is detected. The requirements for gases to be detected with this detector is that they posses a high absorption strength and a characteristic absorption pattern in the wavelength range of the $\mathrm{CO}_{2}$-laser. Inside the photoacoustic cell traces of ET can absorb the laser radiation; the absorbed energy is released into heat, which creates an increase in pressure inside a closed volume. By modulating the laser beam with a mechanical chopper, pressure waves (i.e., sound) are generated and detected with a sensitive miniature microphone. The laser-based photoacoustic detector is able to distinguish between different gases by making use of their wavelength-dependent fingerpint absorption characteristics. ET gas mixtures are sensitively measured by the laser-based photoacoustic detector due to the distinct fingerprint-like spectrum of ET in the $\mathrm{CO}_{2}$-laser wavelength range. By comparing the photoacoustic signals on various laser lines (at which ET has different absorption strenghts) the response of ET can be distinguished from interfering background signals that do not show such an absorption spectrum. The $\mathrm{CO}_{2}$-laser is alternately tuned to the 10P14 $\left(\lambda=948.48 \mathrm{~cm}^{-1}\right)$ and 10P12 $\left(\lambda=951.19 \mathrm{~cm}^{-1}\right)$ laser lines, and at each line the photoacoustic signal is recorded. The absorption coefficients of ET at these wavelengths (see Fig. 2) add specificity to the calculation of the ET concentration. In addition, several measures were taken to prevent 
interfering gases from entering the detection cell in the first place. A sodalime scrubber and a $\mathrm{KOH}$ scrubber were used to remove $\mathrm{CO}_{2}$ from the sample air. $\mathrm{A} \mathrm{CaCl}_{2}$ scrubber was used to decrease the water content of the sample gas. Volatile gases were removed using a liquid nitrogen cooled cryogenic trap $(125 \mathrm{~K})$. The amplitude of the acoustic waves is directly proportional to the concentration of ET in the photoacoustic cell (Harren and Reuss, 1997). The length of the detection cell and the frequency of modulation of the laser are matched, so that the generated sound resonates inside the detection cell. This resonance-effect significantly increases sensitivity. The photoacoustic cell consists of a straight tube, with a small microphone embedded in the middle, a gas inlet, and a gas outlet. The small volume of the detection cell allows a fast response because the sample is not diluted when flowing through the cell. This system has a detection limit for ethylene of 6 ppt. (Harren and Reuss, 1997). Fig. 3 shows the time response of the detection system.

Laser based photoacoustic detectors are able to monitor trace gas concentrations under atmospheric conditions with orders of magnitude better sensitivity than most gas chromatography instrumentation. In addition, they are able to monitor non-invasively and on-line under dynamic conditions (Harren et al., 2000). The photoacoustic instrument used in this study has been developed at the University of Nijmegen specifically for biological applications, in particular for monitoring the production of ethylene by plants (Bijnen et al., 1996; Voesenek et al., 1990).

\section{Subjects}

All volunteers were males between the ages of 20 and 53 years, see also Tab. 1. None of the subjects indicated to have any chronic illnesses, and all were non-smokers. ET levels were measured at rest. The experiments were carried in accordance with the guidelines stated in the 'World Medical Association Declaration of Helsinki-Ethical Principles for Medical Research Involving $\mathrm{Hu}-$ man Subjects' (52nd WMA General Assembly, 2000) and the 'Convention for the Protection of Human Rights and Dignity of the Human Being with regard to the Application of Biology and Medicine: Convention on Human Rights and Biomedicine' (Convention on Human Rights and Biomedicine, 1997) .

\section{Experiments}

Two types of pharmacokinetic experiments were performed. In both, a volunteer breathed from the closed circuit for approximately one hour. In the first type of experiment, a small amount of pure ET (Air Liquide, Eindhoven, The Netherlands) was injected into the system using a syringe, and the de- 
cline of the high initial concentration was monitored. Eleven measurements on 4 subjects were performed in this way, with initial concentrations varying from 0.1-5 ppm (Tab. 1). These measurements allowed examining of the partitioning of ET between body and air. In the second type of experiments the system was flushed with compressed air prior to connecting the volunteer. In this way, a low $(<5 \mathrm{ppb})$ initial concentration was achieved. Eighteen experiments with low initial concentration were performed on 9 subjects in this manner (Tab. 1). These measurements were used to quantitate the endogenous production of ET. In addition to these experiments, we used for our model other experimental data from UV light experiments (Harren et al., 1999). The concentration-time curves of all experiments were simulated using the two pharmacokinetic models.

\section{Analysis}

\section{The two-compartment model}

The two-compartment model used here was taken from literature (Filser, 1992). It assumes the whole body of the organism to function as one well-mixed compartment. A schematic representation of the two-compartment model is given in Fig. 4. The model is described by the two differential equations shown in Eq. 1 and Eq. 2. Here, $V_{1}$ represents the volume of the closed breath circuit (including lung volume), and $V_{2}$ represents the volume of the body of the volunteer. $y_{1}(t)$ and $y_{2}(t)$ represent the ET-concentration in the closed chamber and the body of the subject, respectively. Both compartments are separated by a boundary layer representing the alveolar surface. The exchange of gases through this boundary layer is a first-order process where $k_{12}$ and $k_{21}$ are the exchange constants $\left(\left[\mathrm{h}^{-1}\right]\right)$. The elimination of ET from the body is described by the the metabolic rate constant $k_{\mathrm{el}}\left(\left[\mathrm{h}^{-1}\right]\right)$. Production of ET by the body is described by the rate of endogenous production $d N_{\mathrm{pr}} / d t\left(\left[\mathrm{nmol} \cdot \mathrm{h}^{-1}\right]\right)$.

$$
\begin{aligned}
& V_{1} \frac{d y_{1}(t)}{d t}=V_{2} k_{21} y_{2}(t)-V_{1} k_{12} y_{1}(t) \\
& V_{2} \frac{d y_{2}(t)}{d t}=V_{1} k_{12} y_{1}(t)-V_{2}\left(k_{21}+k_{\mathrm{el}}\right) y_{2}(t)+\frac{d N_{\mathrm{pr}}}{d t}
\end{aligned}
$$

Analysis of the closed chamber measurements starts with fitting a doubleexponential to data from experiments with a high initial concentration (Denk, 1990), see Eq. 3. The curve parameters $\lambda_{1}, \lambda_{2}, C_{1}$ and $C_{2}$ determine the exchange rate constants $k_{12}$ and $k_{21}$ and the metabolic rate constant $k_{\mathrm{el}}$, as shown in Eq. 4, 5 and 6. $C_{3}$ represents a plateau concentration where endogenous production and metabolic clearance are in equilibrium. The value of $C_{3}$ was set to zero in fitting the double-exponential to the data, because 
the effect of endogenous production of ET was considered negligible at high concentrations. After $k_{12}, k_{21}$ and $k_{\text {el }}$ have been determined, $d N_{\text {pr }} / d t$ can be obtained by applying a numerical fit to data from measurements with low initial concentration. The body burden of $\mathrm{EO}$ due to endogenous production of $\operatorname{ET}\left(y_{2}^{\mathrm{EO}}\right)$ is determined according to Eq. 7. In Eq. 7 the sum of $k_{\mathrm{el}}^{\mathrm{EO}}$ and $k_{21}^{\mathrm{EO}}$ was taken from literature (Denk, 1990) and equals 0.999. $F$ represents the fraction of ET that is metabolised to EO and was set to 1 (Denk, 1990). Results for $y_{2}^{\mathrm{EO}}$ therefore represent a worst case scenario. In previous studies (Denk, 1990), $y_{2}^{\mathrm{EO}}$ has been used to asses the risk of cancer resulting from endogenous ET. Such a risk assessment is beyond the scope of this study. Examples of experimental concentration-time curves and corresponding curve fits are given in Fig. 5.

$$
\begin{aligned}
& y_{1}=C_{1} \exp \lambda_{1} t+C_{2} \exp \lambda_{2} t+C_{3} \\
& k_{\mathrm{el}}=-\frac{\lambda_{1} \lambda_{2}\left(C_{1}+C_{2}\right)}{\left(C_{1} \lambda_{1}+C_{2} \lambda_{2}\right)} \\
& k_{12}=\frac{\lambda_{1} \lambda_{2}}{k_{\mathrm{el}}} \\
& k_{21}=-\left(\lambda_{1}+\lambda_{2}+k_{\mathrm{el}}+k_{12}\right) \\
& y_{2}^{\mathrm{EO}}(t)=\frac{k_{\mathrm{el}} y_{2}(t)}{F\left(k_{\mathrm{el}}^{\mathrm{EO}}+k_{21}^{\mathrm{EO}}\right)}
\end{aligned}
$$

In addition to the data from closed chamber measurements, concentrationtime curves were also taken from a previous study (Harren et al., 1999). There, ultraviolet light (UV) was used to induce lipid peroxidation in the skin of human volunteers (UV-measurements). The ET-concentration in exhaled air was measured real-time using a single pass setup. In a single pass experiment, the volunteer inhales from a buffer bag filled with synthetic air containing a low, constant concentration of ET. A small amount of exhaled air is sampled, while contamination with ambient air is minimized. To simulate single pass measurements with the two-compartment model it has to be adjusted so that it deals with inhaling from a low concentration, exhaling into open air, and increased ET production due to irradiation with ultraviolet light. These adjustments are shown in Eq. 8 and 9. In Eq. 8, the breath volume (BV) signifies the amount of air exhaled in litres per hour. To simulate increased ET production in the skin, a new production term $d N_{\mathrm{pr}}^{\mathrm{UV}} / d t$ was placed in the compartment representing the body. The value of $d N_{\mathrm{pr}}^{\mathrm{UV}} / d t$ was determined by fitting simulated concentration-time curves to the data.

$$
y_{1}^{\mathrm{SP}}(t)=\frac{V_{2} k_{21} y_{2}(t)}{B V}
$$




$$
V_{2} \frac{d y_{2}^{\mathrm{SP}}(t)}{d t}=-V_{2}\left(k_{21}+k_{\mathrm{el}}\right) y_{2}(t)+\frac{d N_{\mathrm{pr}}}{d t}+\frac{d N_{\mathrm{pr}}^{\mathrm{uv}}}{d t}
$$

\section{The PBPK model}

The PBPK model used here is an adaptation of an earlier model (Csanády et al., 2000) that links the pharmacokinetics of ET and EO. Compartments related only to EO were omitted. The PBPK model separates the body into a series of biologically and anatomically relevant compartments. All compartments are then connected in anatomical order, based on the blood circulatory system. The model describes uptake via inhalation, endogenous production and metabolism of ET. The model structure is displayed in Fig. 4. Metabolic clearance $(\mathrm{Cl})$, cardiac output $\left(Q_{\text {car }}\right)$, lung volume and alveolar ventilation $\left(A_{\text {alv }}\right)$ were assumed to scale linearly with body surface (Johanson and Filser, 1996), estimated from the volunteers height and weight (Mosteller, 1987). A mathematical description of the model and the physiological parameters used therein can be found elsewhere (Csanády et al., 2000).

Also this model has been adjusted to deal with single pass UV-measurements. When simulating these measurements, the ET-concentrations in exhaled air $\left(C_{\mathrm{exh}}^{\mathrm{SP}}\right)$ and lung blood $\left(C_{\mathrm{pb}}^{\mathrm{SP}}\right)$ are given by equations 10 and 11 . When simulating UV-measurements, the equations describing the vessel rich group and the venous blood now contain a term reflecting production of ET resulting from exposure to UV, see Eq. 12 and 13 .

$$
\begin{aligned}
& C_{\mathrm{exh}}^{\mathrm{sp}}=\frac{\left(C_{\mathrm{ven}}-C_{\mathrm{pb}}\right) \cdot Q_{\mathrm{car}}}{B V} \\
& V_{\mathrm{pb}} \frac{d C_{\mathrm{pb}}^{\mathrm{sp}}}{d t}=-A_{\mathrm{alv}} \frac{C_{\mathrm{pb}}}{\lambda_{\mathrm{b}}}+Q_{\mathrm{car}}\left(C_{\mathrm{ven}}-C_{\mathrm{pb}}\right) \\
& V_{\mathrm{vrg}} \frac{d C_{\mathrm{vrg}}}{d t}=Q_{\mathrm{vrg}}\left(C_{\mathrm{art}}-\frac{C_{\mathrm{vrg}}}{\lambda_{\mathrm{vrg}: \mathrm{b}}}\right)+P \cdot \frac{d N_{\mathrm{pr}}^{\mathrm{uv}}}{d t} \\
& V_{\mathrm{ven}} \frac{d C_{\mathrm{ven}}}{d t}=\sum_{\mathrm{i}} \frac{C_{\mathrm{i}} Q_{\mathrm{i}}}{\lambda_{\mathrm{i}}}-Q_{\mathrm{car}} C_{\mathrm{ven}}+(1-P) \cdot \frac{d N_{\mathrm{pr}}^{\mathrm{uv}}}{d t}
\end{aligned}
$$

\section{Results}

\section{Two-compartment model}

Figure 5 shows three of the measurements with high initial concentration and corresponding curve fits that were used for determining the exchange 
constants $k_{12}, k_{21}$ and the elimination constant $k_{\mathrm{el}}$. Figure 5 also shows three of the measurements with low initial concentration and corresponding curve fits. Pharmacokinetic constants and parameters obtained from the curve fits are given in table 2. Published values (Denk, 1990) for $k_{\mathrm{el}}^{\mathrm{EO}}$ and $k_{21}^{\mathrm{EO}}$ were used to calculate the body burden of EO, resulting from endogenous ET. This gave $Y_{2}^{\mathrm{EO}}=41.8 \pm 5.68 \mathrm{pmol} \cdot \mathrm{kg}^{-1}$.

\section{PBPK model}

Attempts were made to fit our data using published values for all model parameters. Simulations did not agree well with data when published values for the blood:air partition coefficient $\left(\lambda_{\mathrm{b}: \mathrm{a}}\right)$ were used, e.g. 0.15 (Steward et al., 1973) and 0.22 (Csanády et al., 2000) for $\lambda_{\mathrm{b}: \mathrm{a}}$ were used. Therefore, $\lambda_{\mathrm{b}: \mathrm{a}}$ was fitted to measurements with high initial concentration, resulting in $\lambda_{\mathrm{b}: \mathrm{a}} 0.092$ \pm 0.029 . An example is given in Fig. 6 . $\lambda_{\text {b:a }}$ was fixed at this value during fitting of the endogenous production to measurements with low initial concentration. This resulted in $d N_{\mathrm{pr}} / d t=75 \pm 10 \mathrm{pmol} \cdot \mathrm{h}^{-1} \cdot \mathrm{kg}^{-1}$.

UV-measurements (Harren et al., 1999) were simulated by temporarily increasing the endogenous production. Overall, optimal agreement with experimental data was obtained when $60 \%$ of the UV-induced ET-production was located in the venous blood, and $40 \%$ in the compartment representing the vessel-rich group.

\section{Discussion}

\section{Two-compartment model}

The metabolic rate constant in the two-compartment model $k_{\mathrm{el}}=1.24 \pm$ $0.02 \mathrm{~h}^{-1}$ was significantly higher than published earlier, $0.39 \mathrm{~h}^{-1}$ (Denk, 1990).

The body burden of EO resulting from endogenous production is significantly lower than previously published (Denk, 1990). When calculating the body burden upon exposure to $1 \mathrm{ppm}$, our results lead to a $10 \%$ greater body burden of EO than published results (Filser et al., 1992).

When using the model parameters obtained in this study, simulations of UVmeasurements show little agreement with experimental data. This may be due to localization of the UV-induced lipid peroxidation near the richly perfused skin, and the resulting rapid transport to lung tissue. This would mean that a substantial part of the body is bypassed in the ethylene transport, which 
would explain why the whole-body approach fails to simulate the observed concentration-time curves.

\section{PBPK model}

No clear optimum for the metabolic clearance value $C l$ in the PBPK model resulted from fitting sessions, because of the adjustment of the blood:air partition coefficient that was required. Use of a previously published value for $\mathrm{Cl}$ (Csanády et al., 2000) gave good agreement between simulation and measurement.

The PBPK model contains numerous parameters. Some of these parameters are usually determined by curve fitting, namely $d N_{\mathrm{pr}} / \mathrm{dt}$ and $C l$. Other parameters such as partition coefficients are known from in vitro studies and should only be determined by curve fitting as a last resort. Parameters that are independent of the chemical, such as cardiac output and alveolar ventilation were never determined by curve fitting. Following these criteria, efforts were made to simulate experimental data while varying only $d N_{\mathrm{pr}} / d t$ and $C l$. No satisfactory agreement between data and simulations could be found in this manner. It was observed that published values of the blood:air partition coefficient (0.15 (Filser et al., 1992), 0.22 (Csanády et al., 2000)) did not give good agreement between simulation and experiment. The variation in published values and its great impact on simulations suggested adjusting the blood:air partition coefficient. The value for the blood:air partition coefficient (0.092) was obtained by numerical curve fitting. Similar simulations could be obtained by changing the fraction $f$ of inhaled ET that reaches the alveoli from 1.00 to 0.21 . Adjusting the blood-air partition coefficient was considered the more plausible solution. An $f$ smaller than 1 for ET has been reported in rats, and was ascribed to the relatively large size of mucus membranes in the respiratory tract of the rat (Johanson and Filser, 1992).

The best way to include a UV-related production term would be to create a skin compartment and include the production term there. To do this, several physiological parameters have to be known. In the case of skin, the skin-air or skin-blood partition coefficient could not be found in literature. Also, the rate at which ET permeates through the skin could not be found. To avoid including a skin compartment, UV-related ET production was placed in two existing compartments. Part of the ET production was included in the vesselrich group compartment, to represent ET production in richly perfused skin tissue. The rest of the UV-related ET production was placed in the venous blood, to represent ET production in the blood vessels exiting the skin. The fraction of the UV-related production located in each compartment can be adjusted by changing the variable $P$. 
In conclusion, endogenous production values agree well between the twocompartment and the PBPK model, and with previous work at our department (Harren et al., 1999). However, endogenous production from both models is significantly lower than published values based on gas chromatography. Gas chromatography requires accumulation of the gas sample and concentration of the analyte on an adsorbing agent. These steps are not necessary with online photoacoustic detection. Also, in this work measurements lasted one hour, instead of two hours in previous studies. This shorter measurement time is compensated by the larger number of datapoints available for curve fits and the higher sensitivity of laser based detection.

It has been shown that laser photoacoustic detection can be used in combination with the closed chamber technique to study the pharmacokinetics of ethylene, as an alternative to gas chromatography. Other endogenous compounds can also be studied using laser photoacoustic detection, where the sensitivity depends on the characteristics of the lightsource and the absorption spectrum of the compound. The real-time nature and high sensitivity of photoacoustic detection allows investigation of dynamical processes on a short timescale and at low concentrations. It is therefore believed that laser photoacoustic detection can be used with the closed chamber technique as a useful addition to conventional detection schemes.

\section{Acknowledgements}

The authors wish to thank Dr. J.G.C. Lerou from the department of anesthesiology of the UMC St Radboud, Nijmegen, The Netherlands for his help in ensuring the safe use of the closed breath circuit.

\section{References}

Convention for the Protection of Human Rights and Dignity of the Human Being with regard to the Application of Biology and Medicine: Convention on Human Rights and Biomedicine.

52nd WMA General Assembly, 2000. World Medical Association Declaration of Helsinki-Ethical Principles for Medical Research Involving Human Subjects. Edinburgh, Scotland.

Bijnen, F., Reuss, J., Harren, F., 1996. Geometrical optimization of a longitudinal resonant photoacoustic cell for sensitive and fast trace gas detection. Rev. Sci. Instrum. 67, 2914-2923.

Csanády, G., Denk, B., Pütz, C., Kreuzer, P., Kessler, W., Baur, C., Gargas, M., Filser, J., 2000. A physiological toxicokinetic model for exogenous and 
endogenous ethylene and ethylene oxide in rat, mouse and human: Formation of 2-hydroxyethyl adducts with hemoglobin and dna. Tox. Appl. Pharm. $165,1-23$.

Denk, B., 1990. Abschätzung des kanzerogenen risikos von ethylen und ethylenoxid für de menschen durch speziesextrapolation von der ratte unter berücksichtigung der pharmokokinetik. thesis University of Munich.

Esterbauer, H., 1996. Estimation of peroxidative damage. a critical review. Pathology biology 44 (1), 25-28.

Filser, J., 1992. The closed chamber technique-uptake, endogenous production, excretion, steady-state kinetics and rates of metabolism of gases and vapors. Arch. Toxicol. 66, 1-10.

Filser, J., Denk, B., Törnqvist, M., Kessler, W., Ehrenberg, L., 1992. Pharmacokinetics of ethylene in man and body burden with ethylene oxide and hydroxylethylation of hemoglobin due to endogenous and environmental ethylene. Arch. Toxicol. 66, 157-163.

Gelmont, D., Stein, R., Mead, J., 1981. The bacterial origin of breath pentane. Biochem. Biophys. Res. Commun. 102, 932-936.

Harren, F., Berkelmans, R., Kuiper, K., te Lintel Hekkert, S., Scheepers, P., Dekhuizen, R., Hollander, P., Parker, D., 1999. On-line laser photoacoustic detection of ethene in exhaled air as biomarker of ultraviolet radiation of the human skin. Appl. Phys. Lett. 74 (12), 1761-1763.

Harren, F., Cotti, G., Oomens, J., te Lintel Hekkert, S., 2000. Photoacoustic spectroscopy in trace gas monitoring. In Encyclopedia of Analytical Chemistry 3, 2203-2222.

Harren, F., Reuss, J., 1997. Photoacoustic spectroscopy. In Encyclopedia of Applied Physics, Weinheim: V. C. H. 19, 413-435.

Johanson, G., Filser, J., 1992. Experimental data from closed chamber gas uptake studies in rodents suggest lower uptake rate of chemical than calculated from literature values on alveolar ventilation. Archives of Toxicology 66, 291-295.

Johanson, G., Filser, J., 1996. Pbpk model for butadiene metabolism to expoxides: quantitative species differences in metabolism. Toxicology 113 (1-3), 40-47.

Kessler, W., 1987. Untersuchungen zu aminosaure- und proteinoxidation in eisen/ascorbat- und eisen/ascorbat/gsh-systemen hinsintlich der entstehung von kohlwasserstoffen sowie enzyminaktievierungen. Ph.D. thesis.

Kessler, W., Remmer, H., 1990. Generation of volatile hydrocarbons froms amino acids and proteins by an iron/ascorbate/gsh system. Biochem. Pharmacol. 39, 1347-1351.

Kneepkens, C., Legage, G., Roy, C., 1994. The potential of the hydrocarbon breath test as a measure of lipid peroxidation. Free Radical Biologgy and Medicine 17 (2), 127-160.

Lieberman, M., Kunishi, A., Mapson, L., Wardale, D., 1964. Ethylene production from methionine. Biochem. Pharmacol. 97, 449-459.

Mosteller, R., 1987. Simplified calculation of body surface area. New England 
Journal Medicine 317 (17), 1098.

Steward, A., Allott, P., Cowles, A., Mapleson, W.W.Setlow, R., 1973. Solubility coefficients for inhaled anaesthetics for water, oil and biological media. Br. J. Anaesth. 45, 282.

Törnqvist, M., Gustafsson, B., Kautiainen, A., Harms-Ringdahl, M., Granath, F., Ehrenberg, L., 1989. Unsaturated lipids and intestinal bacteria as sources of endogenous production of ethylene and ethylene oxide. Carcinogenesis 10, $39-41$.

Voesenek, L., Harren, F., Bögemann, Blom, C., Reuss, J., 1990. Ethylene production and petiole growth in rumex plants induced by soil water logging: The application of a continuous flow system and a laser-driven intracavity photoacoustic detection system. Plant Physiology 94, 1071-1077. 


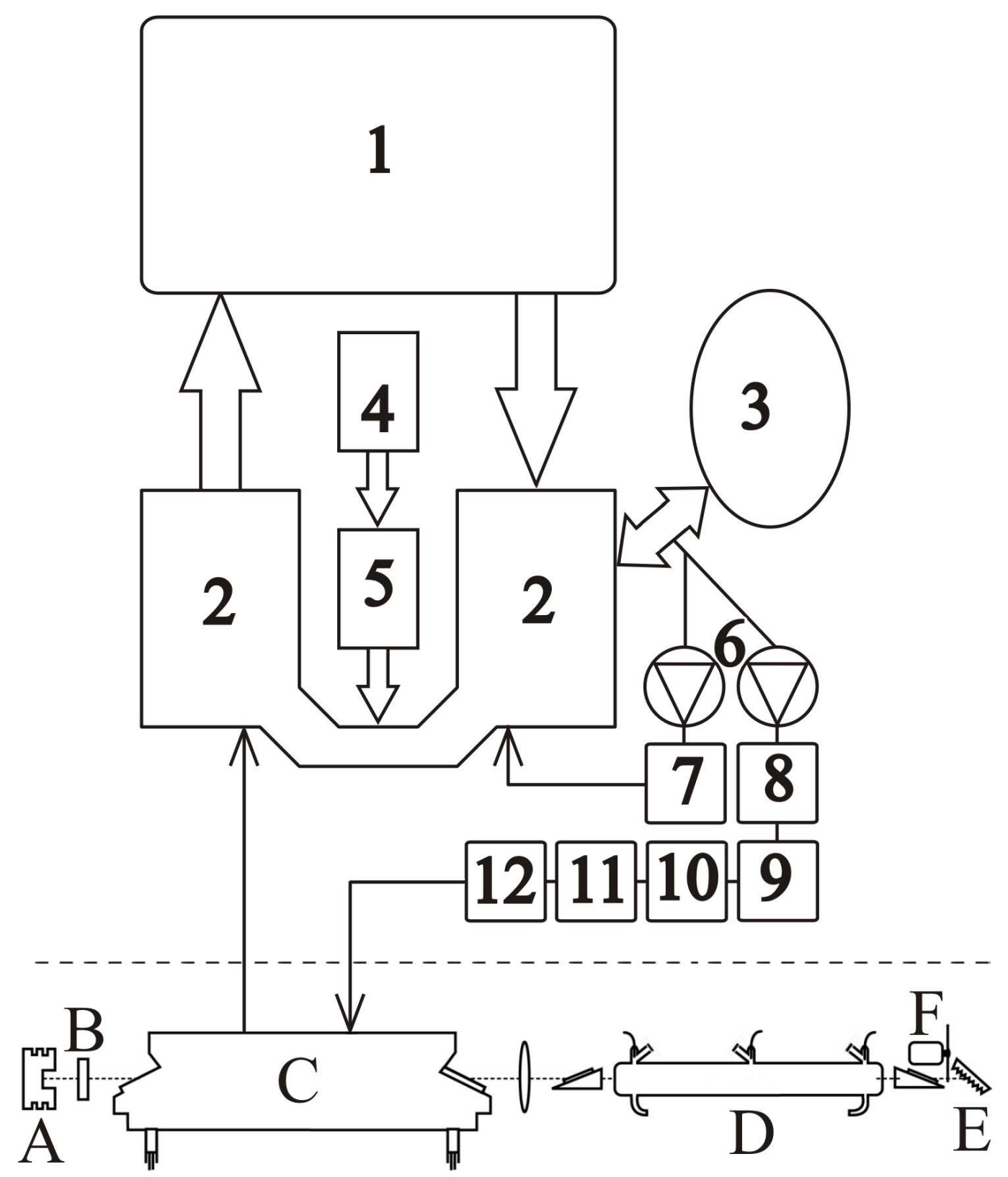

Fig. 1. Experimental setup. The closed gas circuit is formed by: 1) volunteer, 2) sodalime pots, 3) buffer balloon, 4) pressurized $\mathrm{O}_{2}$ bottle, 5) mass flow controller, 6) membrane pumps, 7) URAS 14, 8) sodalime scrubber 9) $\mathrm{KOH}$ scrubber, 10) $\mathrm{CaCl}_{2}$ scrubber, 11) flow controller, 12) cryogenic trap, and C) ET detection cell. The $\mathrm{CO}_{2}$-laser (D) wavelength is determined by the angle of the grating (E). The chopper (F) modulates the laser at the resonance frequency of the photoacoustic detection cell $(\mathrm{C})$, where a microphone records the ethylene signal. A small fraction of the laser light is transmitted by a mirror (B) and quantified using a powermeter (A). 


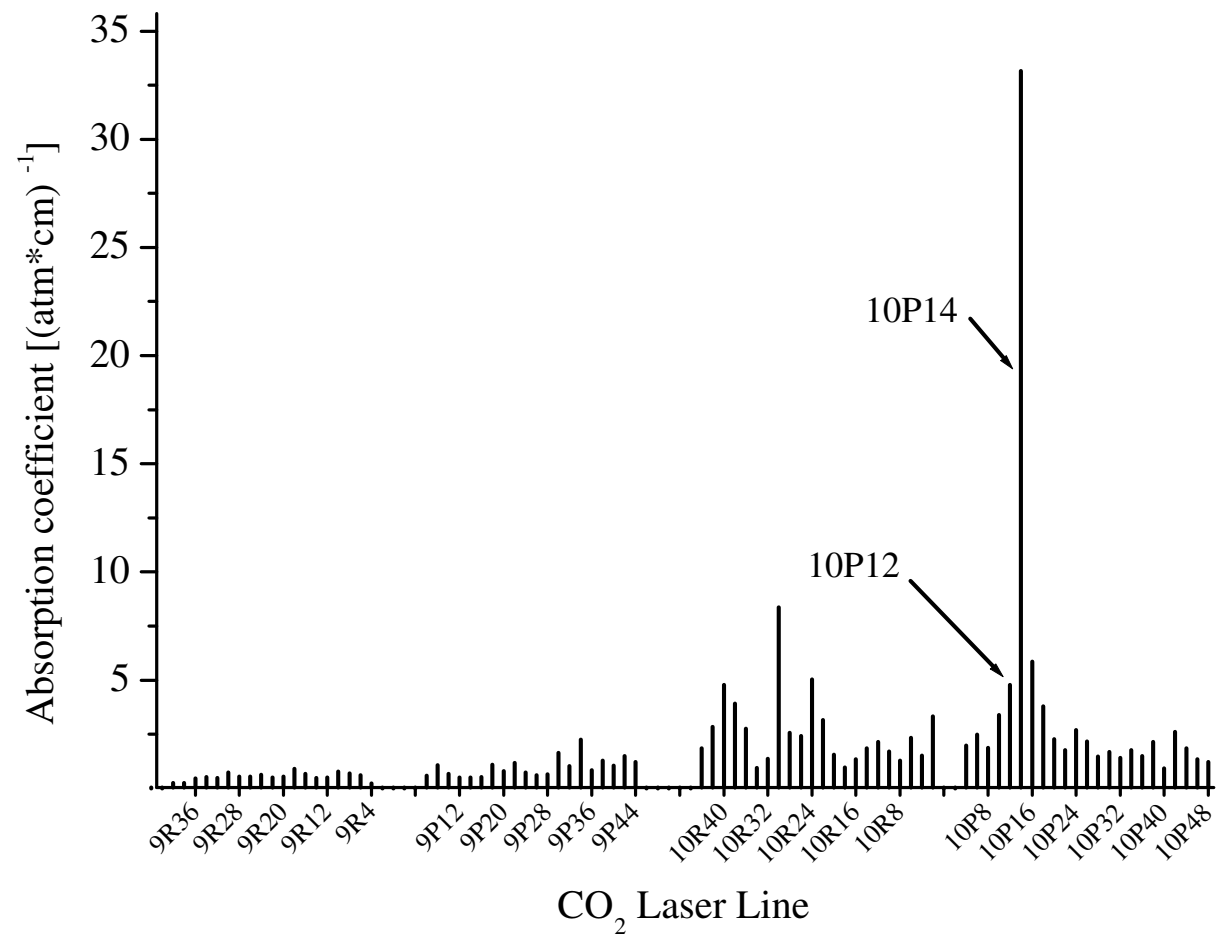

Fig. 2. Absorption coefficients of ethylene at the $\mathrm{CO}_{2}$-laser lines

\begin{tabular}{lllll}
$\begin{array}{llll}\text { Initial } \\
\text { concentration }\end{array}$ & Age $[\mathrm{y}]$ & Height $[\mathrm{cm}]$ & Weight $[\mathrm{kg}]$ & $\mathrm{N}$ \\
\hline Low & $27.8 \pm 10.5$ & $188 \pm 5$ & $81.8 \pm 6.2$ & 9 \\
High & $23.5 \pm 3.8$ & $189 \pm 3$ & $82.0 \pm 3.8$ & 4 \\
\hline
\end{tabular}

Table 1

Volunteers. All values are given in mean + SD 


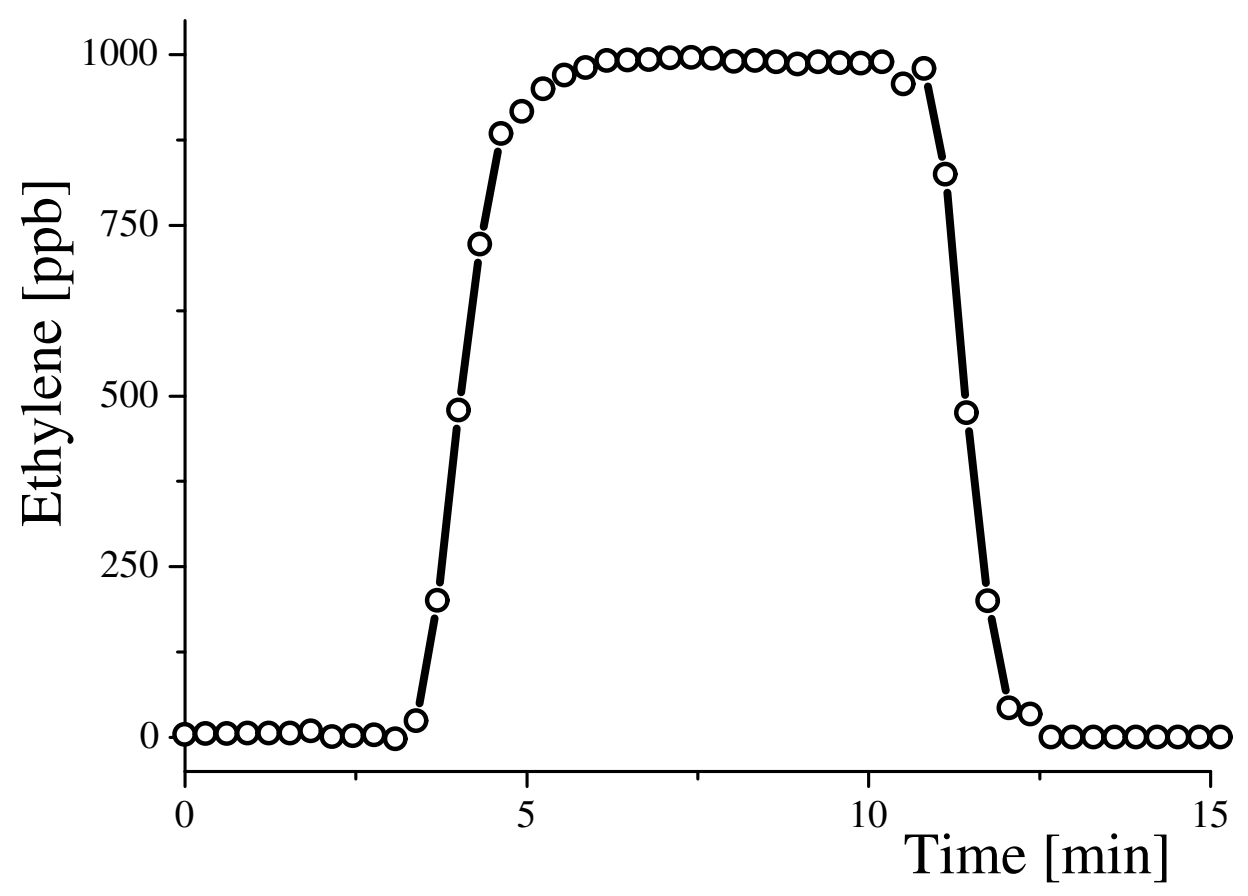

Fig. 3. Time response of the photoacoustic detection system. The detection system was connected to a flow of purified air until $t=3.3 \mathrm{~min}$, when a $3.00 \pm 0.01$ flow of $0.99 \pm 0.01 \mathrm{ppm}$ ethylene was connected. The system was reconnected to purified air at $\mathrm{t}=10.4 \mathrm{~min}$. The doubling time was $12.5 \pm 1.7 \mathrm{sec}$, and the half life was 13.2 $\pm 0.9 \mathrm{sec}$. 


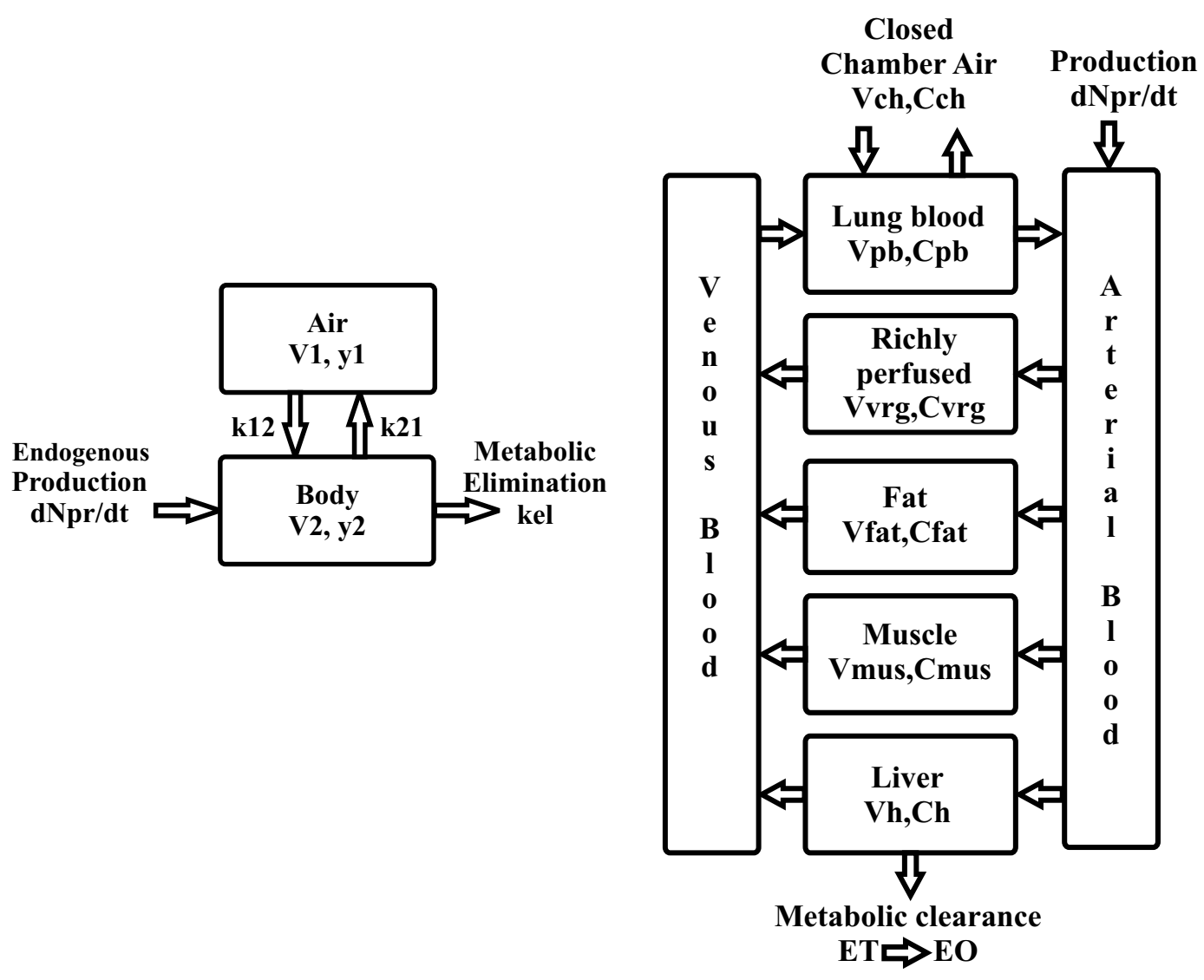

Fig. 4. Schematic representation of the two-compartment model (left) and the PBPK model (right). 



Fig. 5. Metabolism and endogenous production of ET in humans. (A) and (B) Concentration-time courses of ET in the atmosphere of a closed breath circuit, with initial concentrations between 1 and $5 \mathrm{ppm}$. Only 1 out of 5 datapoints is plotted (symbols). The solid lines represent model simulations of the two-compartment model (A), and the PBPK model (B) In (B), the blood:air partition coefficient was equal to 0.092 in all simulations. (C) and (D) Concentration-time courses of ET in the closed breath circuit from measurements with low initial concentration. Only 1 out of 5 datapoints is plotted (symbols). The solid lines represent simulations of the two-compartment model (C) and PBPK model (D). (E) and (F) Concentration-time courses of ET in exhaled air during exposure to ultraviolet light. Only 1 out of 2 datapoints is plotted (symbols). The solid lines respresent simulations of the two-compartment model (E) and PBPK model (F). 


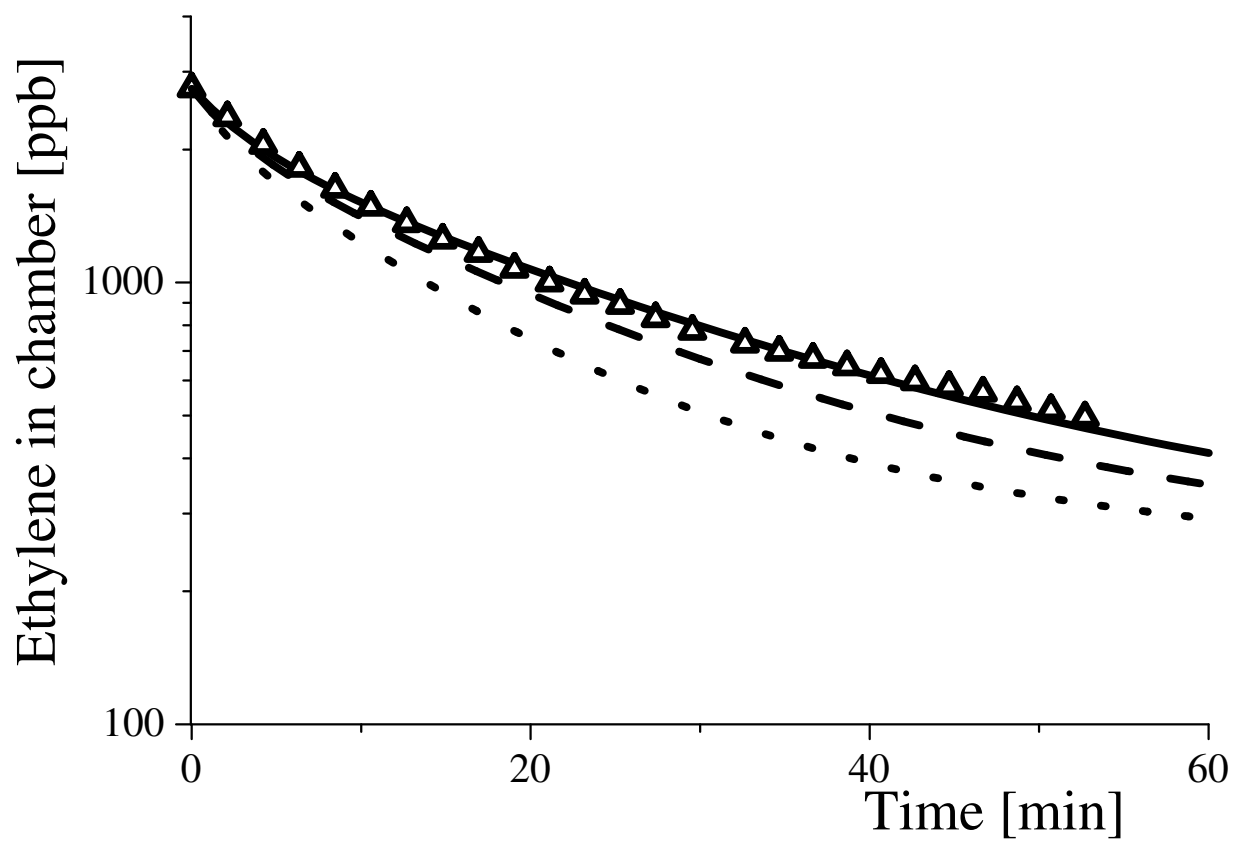

Fig. 6. PBPK model fit to measurement with high initial concentration $(\triangle)$ with published values for $\lambda_{b: a}$ (dotted line:0.22, dashed line:0.15) and the numerically fit value $\lambda_{b: a}=0.092$ (solid line) . Only one out of 5 datapoints is plotted. 


\begin{tabular}{|c|c|c|c|}
\hline Constant & Expression & Value $($ mean $\pm \mathrm{SD})$ & Dimension \\
\hline Clearance of uptake & $k_{12} \cdot V_{1}$ & $20.2 \pm 2.0$ & $1 \cdot h^{-1}$ \\
\hline Rate constant of exhalation & $k_{21}$ & $1.49 \pm 0.09$ & $\mathrm{~h}^{-1}$ \\
\hline Rate constant of metabolism & $k_{\mathrm{el}}$ & $1.24 \pm 0.02$ & $h^{-1}$ \\
\hline Endogenous production rate & $\frac{d N_{\mathrm{pr}}}{d t}$ & $92 \pm 13$ & $\mathrm{pmol} \cdot \mathrm{h}^{-1} \mathrm{~kg}^{-1}$ \\
\hline Parameter & Expression & Value $($ mean $\pm \mathrm{SD})$ & Dimension \\
\hline $\begin{array}{l}\text { Thermodynamic equilibrium } \\
\text { coefficient 'body/air' }\left(K_{\mathrm{eq}}\right)\end{array}$ & $\frac{k_{12} V_{1}}{k_{21} V_{2}}$ & $0.19 \pm 0.02$ & - \\
\hline $\begin{array}{l}\text { Bodyaccumulation factor } \\
\text { 'body/air' at steady state }\left(K_{\mathrm{st}}\right)\end{array}$ & $\frac{k_{12} V_{1}}{\left(k_{21}+k_{\mathrm{el}}\right) V_{2}}$ & $0.11 \pm 0.01$ & - \\
\hline Clearance of exhalation & $k_{21} V_{2} K_{\text {st }}$ & $11.5 \pm 1.3$ & $1 \cdot h^{-1}$ \\
\hline Clearance of metabolism & $k_{\mathrm{el}} V_{2}$ & $86.8 \pm 1.1$ & $l \cdot h^{-1}$ \\
\hline Half-life in organism & $\frac{\ln 2}{\left(k_{\mathrm{el}}+k_{21}\right)}$ & $0.25 \pm 0.02$ & $\mathrm{~h}$ \\
\hline$\%$ metabolised & $\frac{100 \cdot k_{\mathrm{el}}}{\left(k_{\mathrm{el}}+k_{21}\right)}$ & $45 \pm 1$ & $\%$ \\
\hline$\%$ exhaled & $\frac{100 \cdot k_{21}}{\left(k_{\mathrm{el}}+k_{21}\right.}$ & $55 \pm 5$ & $\%$ \\
\hline $\begin{array}{l}\text { Body burden of ET due to } \\
\text { endogenous production of ET }\end{array}$ & $\frac{\frac{d N_{\mathrm{pr}}}{d t}}{\left[\left(k_{\mathrm{el}}+k_{21}\right) V_{2}\right]}$ & $0.048 \pm 0.007$ & $\mathrm{pmol} \cdot \mathrm{ml}^{-1}$ tissue \\
\hline
\end{tabular}

Table 2

Pharmacokinetic constants of ethylene in man $(70 \mathrm{~kg})$. Results are based on eleven measurements on four volunteers. An average value for each volunteer was first obtained, then the final average was calculated. 